\title{
PATIENT SAFETY \\ Functional Contrast-Enhanced CT for Evaluation of Acute Ischemic Stroke Does Not Increase the Risk of Contrast-Induced Nephropathy
}

F.O. Lima

BACKGROUND AND PURPOSE: Concerns have recently grown regarding the safety of iodinated con-

M.H. Lev trast agents used for CTA and CTP imaging. We tested whether the incidence of AN, defined by a

R.A. Levy $\geq 25 \%$ increase in the post-contrast scan creatinine level, was higher among patients with ischemic stroke who underwent a functional contrast-enhanced CT protocol compared with those who had no

G.S. Silva

M. Ebril

É.C. de Camargo

S. Pomerantz

A.B. Singhal

D.M. Greer

H. Ay

iodinated contrast administration.

MATERIALS AND METHODS: The contrast-exposed group consisted of 575 patients with acute ischemic stroke who underwent CTA ( $n=313)$, CTA/CTP $(n=224)$, or CTA/CTP followed by conventional angiography $(n=38$ ) within 24 hours of stroke onset and were consecutively enrolled in a prospective cohort study. The nonexposed group consisted of 343 patients with ischemic stroke, consecutively admitted to the same institution, who did not receive iodinated contrast material. Patients were stratified by baseline eGFR. In the primary analysis, the Fisher exact test was used to compare the incidence of AN between the contrast-exposed and the nonexposed patients at 24,48 , and 72 hours and on a cumulative basis. A secondary analysis compared the incidence of AN in patients who underwent conventional angiography following CTA/CTP versus patients who underwent CTA/CTP only.

W.J. Koroshetz

W.S. Smith

K.L. Furie

RESULTS: The incidence of AN was $5 \%$ in the exposed and $10 \%$ in the nonexposed group $(P=.003)$ Patients who underwent conventional angiography after contrast CT were at no greater risk of AN than patients who underwent CTA/CTP alone (26 patients, $5 \%$; and 2 patients, $5 \%$, respectively; $P=.7$ ).

CONCLUSIONS: Administration of a contrast-enhanced CT protocol involving CTA/CTP and conventional angiography in selected patients does not appear to increase the incidence of CIN.

\begin{abstract}
ABBREVIATIONS: $\mathrm{AN}=$ acute nephropathy; $\mathrm{CIN}=$ contrast-induced nephropathy; $\mathrm{Cr}=$ creatinine $\mathrm{CTA}=\mathrm{CT}$ angiography; CTP $=\mathrm{CT}$ perfusion; eGFR = estimated glomerular filtration rate; IQR $=$ interquartile range; $k \mathrm{~V}(\mathrm{p})=$ kilovolt (peak); $\mathrm{NIH}=$ National Institutes of Health; STOP $=$ Screening Technology and Outcomes Project in Stroke
\end{abstract}

$\mathbf{M}$

ultimodality imaging with MR imaging and CT technology is increasingly being used to aid in the diagnosis and treatment of acute stroke. The advantages of CT over MR imaging include its rapid accessibility, lower costs, shorter scanning-time intervals, and better patient tolerability. CTA can rapidly and noninvasively identify intra- and extracranial vascular stenoses or occlusions. ${ }^{1,2}$ CTP complements the angio-

Received July 3, 2009; accepted after revision September 4.

From the Department of Neurology, (F.O.L., G.S.S., A.B.S., É.C.d.C., D.M.G., H.A., K.L.F.), Stroke Service, Department of Radiology (M.H.L., S.P., R.G.G.), and Department of Neurology, (D.M.G.), Neurocritical Care Service, Massachusetts General Hospital, Boston, Massachusetts; Harvard University (R.A.L.), Cambridge, Massachusetts; Tufts Medical School (M.E.), Boston, Massachusetts; Department of Radiology (H.A.), Martinos Center for Biomedical Imaging, Boston, Massachusetts; National Institute of Neurological Disorders and Stroke (W.J.K.), Bethesda, Maryland; and Department of Neurology (W.S.S.), University of California, San Francisco, San Francisco, California.

Dr. Ay receives grant support from the NIH grant 1-R01-NS059710-01A2. Dr. Singhal receives grant support from the NIH grant R01-NS051412. This work was also supported by the Agency for Healthcare Research and Quality R01 HS011392, NIH P5O NS051343, the American Heart Association-Bugher Foundation, and the Deane Institute for Integrative Research in Atrial Fibrillation and Stroke. We recognize the generous support of the Esther U. Sharp Fund, the Conway Fellowship Fund, the Lakeside Fund, and the Levitt Fund.

Please address correspondence to Fabricio Oliveira Lima, MD, J. P. Kistler Stroke Research Center, Massachusetts General Hospital, 175 Cambridge St, Suite 300, Boston MA, 02114; e-mail: flima@partners.org

Indicates open access to non-subscribers at www.ajnr.org

DOI 10.3174/ajnr.A1927 graphic data, defining vascular territories with reduced perfusion but potentially salvageable tissue. ${ }^{3}$ CTA and CTP identify vascular and tissue targets for reperfusion strategies.

A limitation of using a CT-based imaging platform has been the concern about the safety profile of the iodinated contrast agents used for CTA and CTP imaging. CIN is one of the most common causes of hospital-acquired acute renal failure and is associated with increased morbidity and mortality. ${ }^{4,5}$ To minimize the risk, the American College of Radiology and the European Society of Uroradiology have developed guidelines for the administration of contrast material in patients with renal failure. ${ }^{6}$ A serum creatinine level determination has become a prerequisite for contrast-enhanced studies in many radiology practices.

Variability in the reported incidence of CIN can be explained by disparate definitions, patient populations, contrast doses, routes of administration, and timing of patient follow-up. ${ }^{7,8}$ Many of the published studies examined cardiac patients who underwent angiography and could have had other procedural- or perfusion-related causes of renal insufficiency. ${ }^{9,10}$ Very few studies have been performed in patients with ischemic stroke by using a control group that did not receive contrast material, and none of the studies have stratified patients according to the baseline renal function. ${ }^{11-13}$

Increases in creatinine levels are not uncommon in hospi- 
talized patients. In a recent study, Newhouse et $\mathrm{al}^{14}$ found that $27 \%$ of hospitalized patients with baseline creatinine values between 0.6 and $1.2 \mathrm{mg} / \mathrm{dL}$ and $16 \%$ of those with baseline creatinine values $>2.0 \mathrm{mg} / \mathrm{dL}$ met the definition of having CIN (a 25\% increase in creatinine compared with baseline level) without receiving contrast.

On the basis of these facts, we sought to test whether the incidence of AN was higher among patients with ischemic stroke who underwent CTA, CTP, and conventional angiography compared with those who had no iodinated-contrastagent administered.

\section{Materials and Methods}

\section{Study Population}

The contrast-exposed group consisted of 575 consecutive patients with acute $(<24$ hours from stroke presentation) ischemic stroke enrolled in a prospective cohort study (STOPStroke) at a single academic medical center between March 2003 and June 2005. STOPStroke is an observational study to evaluate the utility of emergency CT/CTA/CTP in patients admitted with suspected acute ischemic stroke. The nonexposed group consisted of 343 consecutive patients with ischemic stroke who did not receive iodinated contrast media for tests or procedures such as CTA and intra-arterial thrombolysis, consecutively presenting to the same institution between September 1999 and June 2000 or between March 2003 and November 2004. Patients already on dialysis treatment were excluded from the study. The study was approved by the institutional review board. Patients provided informed consent for the collection of data.

\section{Neuroimaging Protocol}

Nonenhanced CT and CTA acquisitions were performed according to standard departmental protocols with 8- or 16-section multidetector CT scanners (LightSpeed; GE Healthcare, Milwaukee, Wisconsin). Nonenhanced CT was performed in the transverse plane with the patient in a head holder. Representative sample parameters, with minimal variations between scanners shown as ranges, were as follows: $120-40 \mathrm{kV}(\mathrm{p}), 170 \mathrm{~mA}, 2$-second scanning time, and 5-mm section thickness. Imaging with these parameters was immediately followed by biphasic helical scanning, performed at the same head tilt as nonenhanced CT. CTA was performed after a 25 -second delay (40 seconds for patients in atrial fibrillation) and administration of a nonionic contrast agent at an injection rate of $3 \mathrm{~mL} / \mathrm{s}$ by using a power injector (Medrad Power Injector; Medrad, Indianola, Pennsylvania) via an 18-gauge intravenous catheter. Parameters were $140 \mathrm{kV}(\mathrm{p})$, 220-250 mA, 0.8- to 1.0-second rotation time, 2.5-mm section thickness, $1.25-\mathrm{mm}$ reconstruction intervals, $3.75 \mathrm{~mm}$ per rotation table speed, and 0.75:1 pitch. Images were obtained from the C6 vertebral body level through the circle of Willis. Immediately afterward, a second set of images was obtained from the aortic arch to the skull base. Afterward, source images were reconstructed into standardized maximum-intensity-projection views of the intracranial and extracranial vasculature.

CTP was initiated 5 seconds after the administration of contrast at $7 \mathrm{~mL} / \mathrm{s}$. Four contiguous CT sections were acquired simultaneously every second, during 45-60 seconds. Scanning sections were 5-mmthick. Postprocessing of cerebral blood flow and cerebral blood volume maps was done by using commercially available software (CT Perfusion 3; GE Healthcare). CTA/CTP was the standard protocol for patients with stroke considered within the window for reperfusion therapies $(<12$ hours from stroke-symptom onset). In the 12- to 24-hour window, the decision as to whether to perform CTA or CTA/ CTP was based on clinician preference.

\section{Contrast Agent and Procedures}

All patients in the contrast-exposed group received the nonionic iodinated contrast agent iopamidol (Isovue; Bracco Diagnostics, Princeton, New Jersey) at a dose range from 100 (CTA alone; 313 patients, 54\%) to $140 \mathrm{~mL}$ (CTA and CTP; 224 patients, 39\%). Some patients also underwent transfemoral angiography for intra-arterial thrombolysis after the CTA/CTP (38 patients, 7\%), receiving a larger dose of contrast agent (approximately $230 \mathrm{~mL}$ total). The clinical institutional protocol recommends hydration and pretreatment with $\mathrm{N}$-acetylcysteine in patients considered at "high risk" for contrastinduced nephropathy (eg, patients with diabetes, baseline creatinine level $>1.9 \mathrm{mg} / \mathrm{dL}$ ). Patients in the nonexposed group did not receive iodinated contrast agents.

Patient age, sex, race, and medical history, including diabetes, hypertension, congestive heart failure, and coronary artery disease were collected on all patients through interviews, prospective clinical examinations, and review of the medical records by trained staff. Arterial hypertension was defined as a positive history or antihypertensive treatment. Diabetes mellitus was defined as a positive history or the presence of oral hypoglycemic medication or insulin treatment. Congestive heart failure was defined as a previous positive clinical diagnosis with episodes of dyspnea requiring emergency treatment or inpatient treatment or appropriate medical treatment. Coronary artery disease was defined by a history of myocardial infarction, typical or atypical angina, electrocardiogram evidence of old myocardial infarction, or a history of a cardiac revascularization procedure. All serum creatinine values available at baseline (admission or pre-CTA) and at 24,48 , and 72 hours after the admission were recorded. The eGFR was calculated with the Modification of Diet in Renal Disease Study equation by using the admission creatinine value. ${ }^{15}$ The term "contrastinduced nephropathy" cannot be applied to patients who did not receive contrast agents and implies causality. Therefore, the term "AN" was used to define a $\geq 25 \%$ increase in creatinine from baseline level within 3 days.

Baseline renal dysfunction is an important predictor of a subsequent increase in creatinine levels. ${ }^{8}$ To control for baseline renal function, we performed a stratified analysis based on admission eGFR ( $\geq 60 \mathrm{~mL} / \mathrm{min} / 1.73 \mathrm{~m}^{2}, 30-59 \mathrm{~mL} / \mathrm{min} / 1.73 \mathrm{~m}^{2}$, and $<30 \mathrm{~mL} / \mathrm{min} /$ $1.73 \mathrm{~m}^{2}$ ), according to guidelines of the National Kidney Foundation for classification of chronic kidney disease. ${ }^{16}$

\section{Statistical Analysis}

All statistical analysis was performed by using SPSS software, Version 16.0 (SPSS, Chicago, Illinois). Age, Cr level, and eGFR were analyzed as continuous variables. Data are reported as mean \pm SD or median \pm IQR for continuous variables and as frequency for categoric variables. Differences in age between contrast-exposed and nonexposed groups were compared by using the independent-samples $t$ test. Differences in $\mathrm{Cr}$ level and e-GFR between contrast-exposed and nonexposed groups were compared by using the Mann-Whitney $U$ test. Differences in sex, race, and previous history of hypertension, diabetes, congestive heart failure, and coronary artery disease were assessed by the Fisher exact test. For the primary analysis, all patients who received iodinated contrast agent (including CTA, CTA/CTP, and CTA/CTP plus conventional angiography) were classified under the 


\begin{tabular}{|c|c|c|c|c|}
\hline Characteristics & $\begin{array}{l}\text { Study Population } \\
\qquad(N=918)\end{array}$ & $\begin{array}{l}\text { Contrast-Exposed } \\
\qquad(N=575)\end{array}$ & $\begin{array}{l}\text { Nonexposed } \\
(N=343)\end{array}$ & $P$ \\
\hline Age, mean (yr) & $68 \pm 15$ & $67 \pm 15$ & $70 \pm 14$ & $.005^{\mathrm{a}}$ \\
\hline Sex, \% women & $48 \%$ & $48 \%$ & $48 \%$ & $1.0^{\mathrm{b}}$ \\
\hline Race, \% white & $92 \%$ & $93 \%$ & $91 \%$ & $.3^{\mathrm{b}}$ \\
\hline Hypertension & $60 \%$ & $59 \%$ & $62 \%$ & $.5^{\mathrm{b}}$ \\
\hline Diabetes & $19 \%$ & $17 \%$ & $24 \%$ & $.006^{b}$ \\
\hline Congestive heart failure & $8 \%$ & $6 \%$ & $10 \%$ & $.03^{b}$ \\
\hline Coronary artery disease & $23 \%$ & $22 \%$ & $24 \%$ & $.7^{\mathrm{b}}$ \\
\hline Admission $\mathrm{Cr}(\mathrm{mg} / \mathrm{dL})$, median & $1.07(0.8-1.2)$ & $1.0(0.8-1.1)$ & $1.2(0.8-1.4)$ & $<.001^{\mathrm{c}}$ \\
\hline Admission eGFR $\left(\mathrm{mL} / \mathrm{min} / 1.73 \mathrm{~m}^{2}\right)$, median & $73(56-88)$ & $76(61-89)$ & $68(47-87)$ & $<.001^{\mathrm{c}}$ \\
\hline
\end{tabular}

a Student $t$ test.

b Fisher exact test.

${ }^{\mathrm{c}}$ Mann-Whitney $U$ test.

contrast-exposed group $(n=575)$. Patients who did not receive iodinated contrast agent for any tests or procedures were classified under the nonexposed group $(n=343)$.

We used the Fisher exact test to compare the incidence of AN between the contrast-exposed group and the nonexposed group. A multivariate regression model was used to compare the risk of AN between the exposed and the nonexposed group after adjusting for possible confounders. We also performed a secondary analysis comparing patients who had conventional angiography following CTA/ CTP $(n=38)$ versus patients who underwent CTA and/or CTP alone $(n=537)$. In the secondary analysis, we used the Fisher exact test to compare the incidence of AN between patients who underwent conventional angiography after CTA/CTP and patients who underwent only CTA and/or CTP. The incidence of AN was compared at each time point $(24,48$, and 72 hours) and on a cumulative basis. A 2-sided $P$ value $<.05$ was considered significant. A probability of $4 \%$ for developing CIN was assumed on the basis of previous studies with patients with ischemic stroke. ${ }^{9,17,18}$ We had $80 \%$ power to detect a difference of $3 \%$ in the incidence of AN between patients who received iodinated contrast and patients who did not receive iodinated contrast agents $(\alpha=0.05 ; P<.05)$. On the basis of the same assumptions and a $15 \%$ incidence of $\mathrm{CIN}$ following angiographic procedures, we were $80 \%$ powered to detect a difference of $11 \%$ in the incidence of AN between patients who underwent CTA and/or CTP and patients who underwent CTA/CTP followed by conventional angiography. ${ }^{19}$

\section{Results}

The mean age of the population was $68 \pm 15$ years, $48 \%$ of the patients were women, and $92 \%$ were white. The proportion of patients with conditions that increased the risk of contrast administration, such as diabetes and congestive heart failure, was higher in the noncontrast group (Table 1). Patients in the control group were also older $(P=.004)$ and had high baseline levels of creatinine $(P<.001)$ and, therefore, lower eGFRs at baseline $(P<.001)$.

The overall incidence of AN was 7\% (63 patients). The incidence of AN in contrast-exposed patients was 5\% (28 subjects), which was lower than the $10 \%$ incidence ( 35 subjects) in the nonexposed group $(P=.002)$. Significantly higher incidences of AN were noted in the nonexposed group at 24 and 48 hours, but these were not significantly different by 72 hours (Table 2). In the multivariate regression model, after adjusting for age, sex, admission eGFR, hypertension, diabetes, coronary artery disease, and congestive heart failure, patients in the contrast-exposed group presented a lower risk for developing

\begin{tabular}{|c|c|c|c|}
\hline eGFR/Time Point & $\begin{array}{c}\text { Cases } \\
(\%)\end{array}$ & $\begin{array}{l}\text { Controls } \\
(\%)\end{array}$ & $P^{a}$ \\
\hline \multicolumn{4}{|l|}{ All Patients } \\
\hline 24 Hours (499 cases and 288 controls) & $8(2)$ & $15(5)$ & .007 \\
\hline 48 Hours (428 cases and 232 controls) & $14(3)$ & $18(8)$ & .01 \\
\hline 72 Hours (382 cases and 206 controls) & $19(5)$ & $14(7)$ & .4 \\
\hline $\begin{array}{l}\text { Cumulative incidence (575 cases and } 343 \\
\text { controls) }\end{array}$ & $28(5)$ & $35(10)$ & .003 \\
\hline \multicolumn{4}{|l|}{$\mathrm{eGFR}>60 \mathrm{~mL} / \mathrm{min} / 1.73 \mathrm{~m}^{2}$} \\
\hline 24 Hours (376 cases and 164 controls) & $7(2)$ & $12(7)$ & .004 \\
\hline 48 Hours (320 cases and 133 controls) & $10(3)$ & $10(8)$ & .05 \\
\hline 72 Hours (284 cases and 111 controls) & $14(5)$ & $3(3)$ & .4 \\
\hline $\begin{array}{l}\text { Cumulative incidence (436 cases and } 206 \\
\text { controls) }\end{array}$ & $21(5)$ & $22(11)$ & .01 \\
\hline \multicolumn{4}{|l|}{ eGFR $59-30 \mathrm{~mL} / \mathrm{min} / 1.73 \mathrm{~m}^{2}$} \\
\hline 24 Hours (118 cases and 103 controls) & $1(1)$ & $2(2)$ & .6 \\
\hline 48 Hours (105 cases and 80 controls) & $3(3)$ & $6(8)$ & .2 \\
\hline 72 Hours (95 cases and 76 controls) & $4(4)$ & $7(9)$ & .2 \\
\hline $\begin{array}{l}\text { Cumulative incidence (134 cases and } 115 \\
\text { controls) }\end{array}$ & $6(5)$ & $9(8)$ & .3 \\
\hline \multicolumn{4}{|l|}{$\mathrm{eGFR}<30 \mathrm{~mL} / \mathrm{min} / 1.73 \mathrm{~m}^{2}$} \\
\hline 24 Hours (5 cases and 21 controls) & 0 & $1(5)$ & 1.0 \\
\hline 48 Hours ( 5 cases and 19 controls) & $1(20)$ & $2(11)$ & .4 \\
\hline 72 Hours ( 5 cases and 19 controls) & $1(20)$ & $4(21)$ & 1.0 \\
\hline Cumulative incidence ( 5 cases and 22 controls) & $1(20)$ & $4(18)$ & .9 \\
\hline
\end{tabular}

a Fisher exact test.

AN than patients in the nonexposed group (odds ratio, 0.42; 95\% confidence interval, 0.24-0.71).

Thirty-eight patients underwent conventional angiography following the contrast-enhanced CT. The patients who underwent angiography did not differ significantly from the patients who underwent CTA and/or CTP alone on the basis of age ( $67 \pm 17$ years versus $65 \pm 15$ years, $P=.8)$, sex $(53 \%$ women versus $47 \%$ women, $P=.5$ ), race ( $92 \%$ white versus $93 \%$ white, $P=.7)$, hypertension $(67 \%$ versus $58 \%, P=.4)$, diabetes $(21 \%$ versus $16 \%, P=.4)$, congestive heart failure ( $10 \%$ versus $2 \%, P=.2$ ), coronary artery disease (32\% versus $22 \%, P=.2)$, baseline creatinine level $(0.9 \mathrm{mg} / \mathrm{dL}$; IQR, $0.8-$ 1.2 versus $1.0 \mathrm{mg} / \mathrm{dL}$; IQR, $0.9-1.1 ; P=.9)$ or eGFR $(72 \mathrm{~mL} /$ $\mathrm{min} / 1.73 \mathrm{~m}^{2}$; IQR, $63-84$ versus $76 \mathrm{~mL} / \mathrm{min} / 1.73 \mathrm{~m}^{2}$; IQR, 60-90), respectively. The overall incidence of $\mathrm{AN}$ in both groups was $5 \%(P=.7)$. The incidence of $A N$ at 24,48 , and 72 hours did not differ between the angiography and the CTA/ CTP only group. 


\section{Discussion}

This is the largest reported retrospective cohort study examining the risk of contrast-induced nephropathy in patients with acute stroke. We found that the incidence of AN in the contrast-exposed group did not exceed the incidence of AN in the nonexposed group. In fact, after adjusting for possible confounders, the contrast-exposed group had a lower risk for developing AN than the nonexposed group. The addition of contrast used for conventional angiography after CTA/CTP did not increase the incidence of AN.

Contrast-induced nephropathy is the third most common cause of renal failure, accounting for $11 \%$ of cases of hospitalacquired renal insufficiency. ${ }^{4}$ The most commonly accepted definition of CIN is an increase in creatinine of $\geq 25 \%$ of the baseline value or an absolute increase of $\geq 0.5 \mathrm{mg} / \mathrm{dL}$ in creatinine above the baseline value within $48-72$ hours of exposure to contrast material. ${ }^{8}$ Serum creatinine level typically peaks 3-5 days after contrast administration and returns to baseline within 1-3 weeks. ${ }^{20}$ Several conditions increase the risk for CIN. Pre-existing renal disease with an elevated level of serum creatinine level is the primary risk factor for developing of $\mathrm{CIN}^{8}{ }^{8} \mathrm{~A}$ baseline test of renal function is strongly recommended by the American College of Radiology and European Association of Uroradiologists as a way to assess the risk of CIN. $^{6}$

Expanded use of CT-based multimodal imaging has been constrained by concerns about potential nephrotoxicity, particularly because time pressures in evaluating patients with acute stroke can necessitate making decisions regarding contrast administration in the absence of a baseline creatinine value. Smith et $\mathrm{al}^{1}$ demonstrated that waiting for the baseline creatinine level resulted in lengthy delays (average time, $73.3 \pm 51$ minutes) in patients with acute stroke. Because acute stroke management protocols seek to minimize the time from symptom onset to thrombolytic delivery, any delay in the process of evaluation is detrimental. This study supports the safety of intravenous contrast agents in patients with acute stroke independent of baseline eGFR when standard prophylactic measures are taken (including the use of low-osmolar contrast agents, adequate intravenous hydration, and pretreatment with $N$-acetylcysteine in high-risk patients).

A combination of several mechanisms is thought to be responsible for the development of CIN. Renal vasoconstriction and direct tubular injury are thought to be the main factors. The medullary portions of the kidney are particularly vulnerable to reductions in blood flow, given the long length of the vasa recta, the low levels of partial pressure of oxygen encountered, and the high oxygen requirements of the renal tubules responsible for salt reabsorption. Direct tubular injury by contrast agents may be exacerbated by renal vasoconstriction. ${ }^{21}$ The incidence of CIN might have been overestimated by extrapolating data from cardiology patients who underwent conventional angiography. Local renal hypoxia may be aggravated by other complications frequently found in this population of patients, such as transient reduced cardiac output and perturbations in the pulmonary ventilation-perfusion relationship.

Our data are consistent with the low rates of CIN reported after CTA/CTP in patients with stroke. ${ }^{17,18,22}$ The incidence of CIN might have been overestimated in previous studies though. As many as $27 \%$ of hospitalized patients with baseline creatinine values between 0.6 and $1.2 \mathrm{mg} / \mathrm{dL}$ and $16 \%$ of those with baseline creatinine values of $>2.0 \mathrm{mg} / \mathrm{dL}$ would be included under the definition of CIN (a $25 \%$ increase in creatinine compared with baseline level) without receiving contrast material. Very few studies have used control groups to compare the risk of $\mathrm{AN}$ in patients receiving and not receiving iodinated contrast. ${ }^{12,13}$

Pre-existing renal dysfunction is the most important risk factor for the development of CIN. Although an elevated baseline creatinine level is a marker of pre-existing nephropathy, it is not reliable enough to identify patients at risk for CIN. ${ }^{8}$ Creatinine clearance is the most reliable way to evaluate renal function. Its estimation can be easily performed by using the Modification of Diet in Renal Disease Equation. ${ }^{15}$ The risk of CIN increases as the eGFR falls, particularly below $60 \mathrm{~mL} / \mathrm{min} /$ $1.73 \mathrm{~m}^{2} .{ }^{23}$ Our study is the first retrospective cohort study to stratify on the basis of baseline renal function.

There are limitations to our study. Patients undergoing the CTA/CTP were highly selected, as evidenced by the higher rates of diabetes, congestive heart failure, and renal insufficiency in the control population. We believe that this recapitulates routine clinical practice, however. We did not collect data on compliance with recommendations for renal protection after contrast administration (including intravenous hydration and pretreatment with $\mathrm{N}$-acetylcysteine), though given the acute nature of the studies, hydration was likely the only potential intervention. Because of the low number of cases, the results are not generalizable to patients with severe renal insufficiency (eGFR <30).

\section{Conclusions}

Although, to our knowledge, it is yet to be established that acute CTA and CTP imaging improve stroke outcomes, they are commonly used in evaluating patients with stroke. This study provides additional evidence that contrast administration does not increase the risk of nephrotoxicity, provided standard prophylactic measures are taken.

\section{Acknowledgments}

We sincerely thank the patients who participated and members of the Massachusetts General Hospital Stroke Service who provided invaluable support.

\section{References}

1. Smith WS, Roberts HC, Chuang NA, et al. Safety and feasibility of a CT protocol for acute stroke: combined CT, CT angiography, and CT perfusion imaging in 53 consecutive patients. AJNR Am J Neuroradiol 2003;24:688-90

2. Lev MH, Farkas J, Rodriguez VR, et al. CT angiography in the rapid triage of patients with hyperacute stroke to intraarterial thrombolysis: accuracy in the detection of large vessel thrombus. J Comput Assist Tomogr 2001;25: $520-28$

3. Wintermark M, Reichhart M, Thiran JP, et al. Prognostic accuracy of cerebral blood flow measurement by perfusion computed tomography, at the time of emergency room admission, in acute stroke patients. Ann Neurol 2002;51: 417-32

4. Nash K, Hafeez A, Hou S. Hospital-acquired renal insufficiency. Am J Kidney Dis 2002;39:930-36

5. Gruberg L, Mintz GS, Mehran R, et al. The prognostic implications of further renal function deterioration within $48 \mathrm{~h}$ of interventional coronary procedures in patients with pre-existent chronic renal insufficiency. J Am Coll Cardiol 2000;36:1542-48

6. Morcos SK, Thomsen HS, Webb JA. Contrast-media-induced nephrotoxicity: 
a consensus report-Contrast Media Safety Committee, European Society of Urogenital Radiology (ESUR). Eur Radiol 1999;9:1602-13

7. McCullough PA, Adam A, Becker CR, et al. Epidemiology and prognostic implications of contrast-induced nephropathy. Am J Cardiol 2006;98:5K-13K

8. Mehran R, Nikolsky E. Contrast-induced nephropathy: definition, epidemiology, and patients at risk. Kidney Int Suppl 2006:S11-15

9. Rao QA, Newhouse JH. Risk of nephropathy after intravenous administration of contrast material: a critical literature analysis. Radiology 2006; 239:392-97

10. Wyman RM, Safian RD, Portway V, et al. Current complications of diagnostic and therapeutic cardiac catheterization. J Am Coll Cardiol 1988;12:1400-06

11. Langner S, Stumpe S, Kirsch M, et al. No increased risk for contrast-induced nephropathy after multiple CT perfusion studies of the brain with a nonionic, dimeric, iso-osmolal contrast medium. AJNR Am J Neuroradiol 2008;29: 1525-29

12. Cramer BC, Parfrey PS, Hutchinson TA, et al. Renal function following infusion of radiologic contrast material: a prospective controlled study. Arch Intern Med 1985;145:87-89

13. Heller CA, Knapp J, Halliday J, et al. Failure to demonstrate contrast nephrotoxicity. Med J Aust 1991;155:329-32

14. Newhouse JH, Kho D, Rao QA, et al Frequency of serum creatinine changes in the absence of iodinated contrast material: implications for studies of contrast nephrotoxicity. AJR Am J Roentgenol 2008;191:376-82

15. Levey AS, Bosch JP, Lewis JB, et al. A more accurate method to estimate glomerular filtration rate from serum creatinine: a new prediction equation-
Modification of Diet in Renal Disease Study Group. Ann Intern Med 1999;130:461-70

16. National Kidney Foundation. K/DOQI clinical practice guidelines for chronic kidney disease: evaluation, classification, and stratification. Am J Kidney Dis 2002;39(2 suppl 1):S1-266

17. Krol AL, Dzialowski I, Roy J, et al. Incidence of radiocontrast nephropathy in patients undergoing acute stroke computed tomography angiography. Stroke 2007;38:2364-66

18. Hopyan JJ, Gladstone DJ, Mallia G, et al. Renal safety of CT angiography and perfusion imaging in the emergency evaluation of acute stroke. AJNR Am J Neuroradiol 2008;29:1826-30. Epub 2008 Aug 21

19. McCullough PA, Wolyn R, Rocher LL, et al. Acute renal failure after coronary intervention: incidence, risk factors, and relationship to mortality. Am J Med 1997;103:368-75

20. McCullough PA, Sandberg KR. Epidemiology of contrast-induced nephropathy. Rev Cardiovasc Med 2003;4(suppl 5):S3-9

21. Persson PB, Hansell P, Liss P. Pathophysiology of contrast medium-induced nephropathy. Kidney Int 2005;68:14-22

22. Josephson SA, Dillon WP, Smith WS. Incidence of contrast nephropathy from cerebral CT angiography and CT perfusion imaging. Neurology 2005;64:1805-06

23. Solomon R, Deray G. How to prevent contrast-induced nephropathy and manage risk patients: practical recommendations. Kidney Int Suppl 2006: S51-53 